\title{
Chris Heyde's Work in Probability Theory, with an Emphasis on the LIL
}

\author{
Ross Maller*,1
}

In one of his first few papers, [M5], Chris gives necessary and sufficient conditions for the convergence of the series

$$
\sum_{n=1}^{\infty} n^{k} P\left(S_{n} \leq x\right), \quad-\infty<x<\infty
$$

for integers $k=0,1,2, \ldots$, and for a similar series with $n^{k}$ replaced by $e^{r n}$, where $r>0$. Here $S_{n}=X_{1}+X_{2}+\cdots+X_{n}$ is a random walk comprised of independent and identically distributed summands $\left(X_{i}\right)_{i=1,2, \ldots, n}$. Series of the kind in (1) are fundamental objects in renewal theory. Under the assumptions $E|X|<\infty$ and $E X>0$ ( $X$ is any random variable having the same distribution as the $\left.X_{i}\right)$, Chris shows that the series in (1) converges (for the designated value of $k$ ) if and only if $E\left|X^{-}\right|^{k+2}$ is finite, and the series with $n^{k}$ replaced by $e^{r n}$ converges for some $r>0$ if and only if $X^{-}$has an analytic characteristic function, or, equivalently, if $E e^{-\theta X}$ is finite for all $\theta$ in an interval $[0, K]$, for some $K>0$. $\left(X^{-}\right.$is defined as $\min (0, X)$.) Thus, the series in (1) converges if and only if the negative tail of the distribution of the $X_{i}$ is not too heavy. Two aspects are worth noting; first, the $X_{i}$ are allowed to take either sign (early work in renewal theory restricted $S_{n}$ to be a "renewal process", i.e., comprised of non-negative summands); and, secondly, Chris obtains complete characterisations of the convergence of the series, subject only to the $X_{i}$ having a finite and positive expectation. Only much later was this latter restriction relaxed, in connection with (1) (in Kesten and Maller (1996)).

In a follow-up paper to [M5], Chris in [M6] extends the study to look at the magnitudes (rather than just the convergence or not) of the series in (1) (and with $n^{k}$ replaced by $e^{r n}, r>0$ ), considered as functions of $x$. Actually, slightly more general series of the form $\sum_{n} a_{n} P\left(S_{n} \leq x\right)$, where $a_{n}>0$ is a deterministic sequence satisfying a certain regularity condition (related to regular variation), are studied. If $k$ is the "index" of the sequence (the least real $k$ such that $a_{n}=O\left(n^{k}\right)$ as $n \rightarrow \infty$ ) then, when it converges, the asymptotic behaviour of $\sum_{n} a_{n} P\left(S_{n} \leq x\right)$, as $x \rightarrow \infty$, can be specified in terms of the asymptotic behaviour of $a_{n}$. Another theorem shows that the same result holds with $S_{n}$ replaced by the maximal sum, $\max _{1 \leq j \leq n} S_{j}$. The latter result is closely connected with the large sample behaviour (as $\left.x \rightarrow \infty\right)$ of moments of the first passage time, $M(x):=\inf \left\{n \geq 1: S_{n}>x\right\}, x>0$; again, an object of primary interest in renewal theory and its applications.

[M5] and [M6] deal with the convergence of series of probabilities. A very natural next step, given such a convergence, is to apply the Borel-Cantelli lemma and make some kind of statement about the a.s. behaviour of the random walk (where "a.s." stands for "almost surely"). [M6] contains some information on the a.s. convergence of $\max _{1 \leq j \leq n} S_{j}$, analogous to the Marcinkiewicz-Zygmund strong law for $S_{n}$, but Chris soon turned his attention to the more delicate issue of the law of the iterated logarithm

\footnotetext{
*This research was partially supported by ARC grant DP0664603.

${ }^{1}$ Centre for Mathematical Analysis, and School of Finance and Applied Statistics, Australian National University, Canberra, ACT, e-mail: Ross.Maller@anu.edu.au
} 
(LIL). Hartman and Wintner (1941) had given this in its ultimate form: provided the increments of the random walk have finite variance $\sigma^{2}$ and expectation 0 , we have

$$
-1=\liminf _{n \rightarrow \infty} \frac{S_{n}}{\sigma \sqrt{2 n \log \log n}}<\limsup _{n \rightarrow \infty} \frac{S_{n}}{\sigma \sqrt{2 n \log \log n}}=+1 \text {, a.s. }
$$

As part of his deep investigations into functional versions of the LIL (of which, more below), Strassen (1966) proved a converse: (2) can hold only if $S_{n}$ has finite variance $\sigma^{2}$ and expectation 0 . Strassen's proof relied on a representation of Skorokhod, whereby $S_{n}$ has the same distribution as a Brownian motion evaluated at a certain sequence of random times. Chris, in [M19], gives a completely different proof of the converse result by modifying a clever and highly technical method developed just previously by Stone (1966), to investigate recurrence properties of $S_{n} / \sqrt{n}$.

This is Chris's first foray into LIL territory. Prior to this, however, in [M18], Chris proves an interesting result which will have some significant repercussions later. He is aware of course, that central limit theorems with remainder (covered in Peter Hall's article in the present volume), can prove useful in establishing LIL and related results. In [M9] and [M27], Chris has already made contributions to various aspects of the convergence theory of random walks to of stable laws, that is, to situations where the normed and centered sum converges in distribution, but not to the normal distribution. [M18] proves the interesting result, that, for any such walks, the "large deviation" probability, $P\left(\left|S_{n}\right|>x_{n}\right)$, where $x_{n}$ is a deterministic sequence such that $S_{n} / x_{n} \stackrel{P}{\rightarrow} 0$, must satisfy

$$
0<\liminf _{n \rightarrow \infty} \frac{P\left(\left|S_{n}\right|>x_{n}\right)}{n P\left(|X|>x_{n}\right)} \leq \limsup _{n \rightarrow \infty} \frac{P\left(\left|S_{n}\right|>x_{n}\right)}{n P\left(|X|>x_{n}\right)}<\infty
$$

(and the same with $n P\left(|X|>x_{n}\right)$ replaced by $P\left(\max _{1 \leq k \leq n}\left|X_{k}\right|>x_{n}\right)$ ). More precisely, in [M18] Chris proves a much more general result than this: (3) holds provided $S_{n}$ is not in the domain of partial attraction of the normal law; that is, provided no subsequence of $\left(S_{n}\right)$, after norming and centering, converges in distribution to the normal distribution.

This thread is taken up again in [M23], but now applied directly to "generalised iterated logarithm results". This terminology is used to describe a result of the form

$$
0<\limsup _{n \rightarrow \infty} \frac{\left|S_{n}\right|}{b_{n}}<\infty, \text { a.s. }
$$

where now the $\sqrt{n \log \log n}$ norming used in (2) is replaced by a more general deterministic sequence, $b_{n}>0$. Chris shows that if $\lim \sup _{n \rightarrow \infty}\left|S_{n}\right| / b_{n}<\infty$ a.s. for such a $b_{n}$, and $S_{n}$ is not in the domain of partial attraction of the normal law, then it must be the case that $\lim _{n \rightarrow \infty}\left(S_{n}-\operatorname{med}\left(S_{n}\right)\right) / b_{n}=0$ a.s., where $\operatorname{med}\left(S_{n}\right)$ is a median of $S_{n}$. Thus, if for example the $X_{i}$ are symmetrically distributed around zero, a generalised LIL as in (4) can hold only if $S_{n}$ is in the domain of partial attraction of the normal law. This result was published soon after a closely related one of Rogozin (1968); Chris was at the cutting edge of a quite "hot" area of research into understanding the law of the iterated logarithm, and its generalisations.

The results just cited might seem obscure or even cryptic, since the class of random walks belonging to the domain of partial attraction of the normal is extremely narrowly defined; but not so-they provide the key for Kesten (1972) to prove the most general possible form of the LIL: (4) holds, after allowing certain kinds of centering, if and only if $S_{n}$ is in the domain of partial attraction of the normal law.

Papers on the LIL continue to appear even in the 2000's, showing that the subject retains its appeal and vigour. In Kesten and Maller (2004), Harry Kesten and I reconsidered, and extended somewhat, Chris's 1968 and 1969 papers - a long lead time from when I first read them. 
After [M6] and [M23], as the next logical extension of his research, Chris turns his attention to functional limit laws and invariance principles. The note in [M25] gives a rate of convergence result for functionals of the interpolated or "broken line" process $\xi_{n}(t)$ defined by: $\xi_{n}(t)=S_{k} /(\sigma \sqrt{n})$ if $t=k / n$; and

$$
\xi_{n}(t)=\xi_{n}(k / n)+n\left(\xi_{n}((k+1) / n)-\xi_{n}(k / n)\right)(t-k / n),
$$

if $k / n \leq t \leq(k+1) / n, 1 \leq k \leq n, n=1,2, \ldots$. Here the $X_{i}$ have mean 0 and finite variance $\sigma^{2}$, and convergence is to the corresponding functional of Brownian motion.

The paper [M25] deals with a weak invariance principle. Chris is also concerned with strong invariance principles. Rather than work these out just for random walks, however, his research takes a notable new direction, broadening its range of applicability tremendously, by considering limit laws for martingales. In collaboration with long-time colleagues Peter Hall and Bruce Brown, and (at the time) PhD student David Scott, Chris publishes a series of papers, and a book, that do much to demonstrate and publicise the possible applications of martingales.

The first step, as for random walks, is to look at rates of convergence to normality. Heyde and Brown in [M35] do this in a very general way. The normalisation of the martingale is by its (deterministic) standard deviation. A uniform bound for the difference between the cumulative distribution function (cdf) of the normalised martingale, and the standard normal cdf, is given in terms of the conditional variances of the increments and their moments of order great than 2 . The method of proof is via the Skorokhod representation, as in Strassen (1967).

Next, we have an iterated logarithm result in [M48]. The main theorem in this paper is a direct analogue of the classical LIL in (2), but where the summands are now required only to form a stationary martingale difference sequence, satisfying a kind of strong law condition on their conditional variances. This provides a generalisation of a slightly earlier result of Stout (1970), who required that the summands be ergodic, and used a random normalisation of the martingale in terms of the conditional variances of the increments. Its usefulness is demonstrated in [M48] by an application, giving a LIL for autoregressive processes.

Heyde and Scott in [M51] carry on this line of research by giving first a strong invariance principle for martingales, then generalising it to a wide class of processes with stationary increments. The resulting theorems are versions of the Strassen (1964) functional law for the interpolated process, whereby its almost sure cluster set is shown to be precisely the class of absolutely continuous functions $\xi(t)$ on $[0,1]$, with $\xi(0)=0$ and $\int_{0}^{1}(\mathrm{~d} \xi(t) / \mathrm{d} t)^{2} \mathrm{~d} t \leq 1$. The martingale difference sequence in [50] is not in general required to be stationary, so Lindberg-type conditions are imposed. The norming for the martingale is of the form $\sqrt{2 s_{n} \log \log s_{n}}$, where $s_{n}^{2}$ is the variance of the martingale. Thus, we have a constant norming LIL and invariance principle. By contrast, Strassen's (1964) functional law uses a random norming. Chris in [M52] applies his results to get a theorem for stationary linear processes, in a somewhat similar way as he did in [M48], for autoregressive processes.

The threads are drawn together in Hall and Heyde [M68], where the previous constant and random norming versions of the LIL and functional law are replaced by a more general random norming version. The Strassen law is shown to hold when the norming is of the form $W_{n} \sqrt{2 \log \log W_{n}}$, where $W_{n}^{2}$ is asymptotic to a conditional variance of the martingale increments, truncated in a certain (in general, random) way. The resulting theorem is, as the title of the paper suggests, a unification of previous results which can be obtained from it as special cases.

[M72] gives a further application of LIL invariance principles, this time using a clever trick of Barbour (1974) to transfer both weak and strong laws for martingales to convergent tail series of martingale difference sequences. An application to the Pólya urn scheme is given.

Finally in this section, we have [M74], again demonstrating Chris's mastery of a wide range of problem areas, by applying his martingale LIL to obtain an a.s. upper bound for the Hawkins (1958) random sieve. This is a probabilistic device which "produces a random sequence with asymptotic properties similar in many ways to the primes". This neat paper turns us full circle to Feller (1945), who drew attention in the introduction to his paper to an intimate connection between number-theory 
and the law of the iterated logarithm, in respect to "a problem in Diophantine approximations treated by Hardy and Littlewood (1914)". The particular stream of Chris's research outlined in this section has followed classical lines, but in thoughtful, innovative and useful ways.

\section{References}

[1] Barbour, A.D. (1974) Tail sums of convergent series of independent random variables. Proc. Camb. Phil. Soc. $75,361-364$.

[2] Feller, W. (1945) The fundamental limit theorems in probability. Bull. Amer. Math, Soc., 800-832.

[3] Hardy, G.H. and Littlewood, J.E. (1914) Some problems of Diophantine approximation, Acta Math. 37, 155-239.

[4] Hartman, P. and Wintner, A. (1941) On the law of the iterated logarithm, Amer. J. Math., 63, 169-176.

[5] Hawkins, D. (1958) The random sieve. Math. Mag. 31, 1-3.

[6] Kesten, Harry (1972) Sums of independent random variables-without moment conditions. Ann. Math. Statist., 43, 701-732.

[7] Kesten, Harry, and R. A. Maller (1996) Two renewal theorems for general random walks tending to infinity. Prob. Theor. Rel. Fields 106, (1996), 1-38.

[8] Harry Kesten and R. A. Maller (2004) The effect of trimming on the law of the iterated logarithm. In: Stochastic Methods and Their Applications: Papers in Honour of Chris Heyde, Eds J. Gani and E. Seneta, J. Appl. Prob. Special Vol. 41A, (2004), 253-272.

[9] Rogozin, B.A. (1968) On the existence of exact upper sequences. Theor. Prob. Appl., 13, 667-672.

[10] Stout, W.F (1970) The Hartman-Wintner law of the iterated logarithm for martingales. Ann. Math. Statist., $41,2158-2160$.

[11] Strassen, V. (1964) An invariance principle for the law of the iterated logarithm. Z. Wahrscheinlichkeitstheorie und Verw. Gebiete 3, 211-226.

[12] Strassen, V. (1967) Almost sure behaviour of sums of independent random variables and martingales. Proc. V Berk. Symp. Math. Statist. Prob., 2, 315-343. Univ. of Calif. Press. 